\title{
The association of vascular endothelial growth factor receptor-1 with the risk of cancer progression following radical prostatectomy
}

\author{
KAILI MAO $^{1}$, PHILIPPE CAMPARO ${ }^{2}$, CECILE BADOUAL ${ }^{3}$, MICHAËL PEYROMAURE ${ }^{1}$, \\ NICOLAS BARRY DELONGCHAMPS ${ }^{1}$, ANNICK VIEILLEFOND ${ }^{4}$ and ANH-TUAN DINH-XUAN ${ }^{5}$ \\ ${ }^{1}$ Department of Urology, Cochin hospital, 27, rue du Faubourg Saint-Jacques, 75014; ${ }^{2}$ Department of Pathology, \\ Val de Grâce hospital, 74 Boulevard Port Royal, 75005; ${ }^{3}$ Department of Pathology, European-Georges \\ Pompidou hospital, 20 rue Leblanc, 75015; Departments of ${ }^{4}$ Pathology and ${ }^{5}$ Physiology, \\ Cochin hospital, 27, rue du Faubourg Saint-Jacques, 75014 Paris, France
}

Received July 6, 2007; Accepted September 26, 2007

\begin{abstract}
In the current study, we analysed the prognostic value of vascular endothelial growth factor receptor-1 (VEGFR-1) in clinically-localized prostate cancer (PCa). Forty patients who had undergone radical prostatectomy (RP) for clinically-localized PCa were included. Two groups were compared: 17 patients who experienced cancer progression following RP (group 1) and 23 patients who remained free of recurrence after intervention (group 2). Paraffin-embedded sections obtained from the RP specimens of the 40 patients were used to build tissue microarrays. The expression of VEGFR-1 was examined in the RP specimens using immunohistochemistry and was compared between the groups of patients. The two groups had similar tumor characteristics in terms of PSA, Gleason score and pathological stage of cancer. The median intensity score of VEGFR-1 expression was significantly higher in pT3 tumors than in pT2 tumors. Nevertheless, the intensity scores of VEGFR-1 expression were similar in the two groups of patients. Our results suggest that VEGFR-1 expression is not associated with the risk of cancer progression following RP. Therefore, VEGFR-1 may not be of prognostic value in clinically-localized PCa.
\end{abstract}

\section{Introduction}

Vascular endothelial growth factor (VEGF) plays a significant role in angiogenesis. To date, five forms of VEGF have been

Correspondence to: Dr Michaël Peyromaure, Service d'Urologie, Hôpital Cochin, 27, rue du Faubourg Saint-Jacques, 75014 Paris, France

E-mail: michael.peyromaure@nck.aphp.fr

Key words: prostate cancer, angiogenesis, vascular endothelial growth factor, receptor, radical prostatectomy identified: VEGF-A, -B, -C, -D and -E. VEGF-A induces differentiation and proliferation of the endothelial cells of blood vessels. It is involved in the growth of many types of tumors, including prostate cancer (PCa) (1). The expression of VEGF-A in prostatic tissue is associated with the presence of cancer and is higher in metastatic PCa than in localized PCa (2). Some studies have shown that the expression of VEGF-A in PCa tissue is associated with the outcome of patients. Furthermore, there is a correlation between circulating levels of VEGF-A and the stage of PCa. For example, Jones et al found, in a series of 78 men (16 with localized PCa, 32 with metastatic $\mathrm{PCa}, 9$ with benign prostatic hyperplasia and 21 healthy controls), that serum VEGF-A levels were significantly higher in patients with hormone-refractory PCa than in other groups (3). These findings give strong evidence that VEGF-A in prostatic tissue, and in blood, has a prognostic value in $\mathrm{PCa}$.

VEGFR-1 is one of the receptors for VEGF-A. The functions of VEGFR-1 remain unclear. Preliminary in vivo studies suggested that VEGFR-1 was a negative regulator of VEGF activity, however, further investigations reached opposite conclusions (1). Clinical data regarding the expression of VEGFR-1 in PCa are lacking. Therefore, the role of this receptor in $\mathrm{PCa}$ is unknown. In the current study, we tried to determine the prognostic value of VEGFR-1 in patients treated for clinically-localized PCa. We compared VEGFR-1 expression in the radical prostatectomy (RP) specimens of two groups of patients: one group of patients who experienced cancer progression following RP and another group of patients who remained free of recurrence after intervention.

\section{Materials and methods}

Study population. Forty patients were included. All the patients underwent RP for PCa between 1994 and 2004. Before RP, a bone scintigraphy and a pelvic CT scan were performed in all cases and no patient had clinical or radiological evidence of lymph node involvement or bone metastases at the time of surgery. A standard lymphadenectomy was performed at the 
A

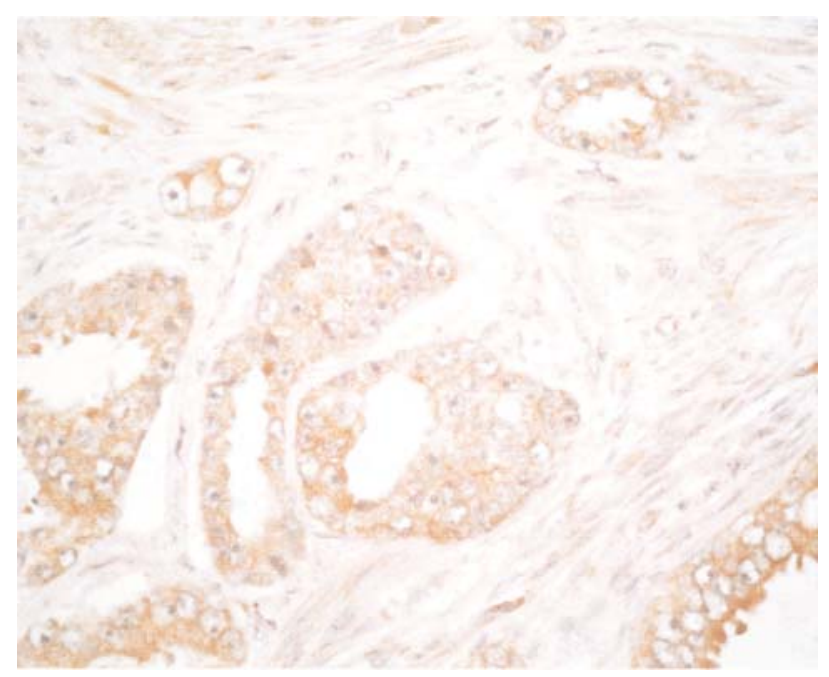

B

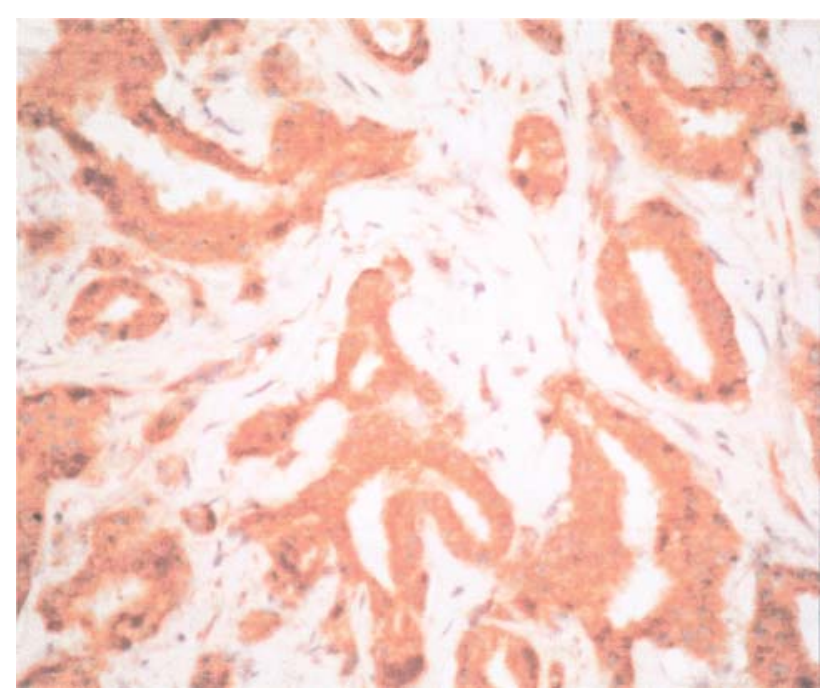

Figure 1. (A) Case of PCa with low VEGFR-1 expression (intensity score) $\mathrm{x} 400$. (B) Case of PCa with high VEGFR-1 expression (intensity score) $\mathrm{x} 400$.

time of RP, and no patient had lymph node involvement. After RP, 17 patients (group 1) experienced biological recurrence and developed bone metastases. Of these patients, 10 received salvage radiotherapy while 7 received hormone therapy at the time of biological recurrence. Bone metastases were diagnosed by scintigraphy in all cases. The median interval between RP and the occurrence of bone metastases was 48 months (range from 16 to 73 ). The remaining 23 patients (group 2) did not experience biological recurrence following intervention. None of them received adjuvant therapy. All the patients in group 2 had an undetectable PSA at the time of analysis, with a median follow-up of 112 months (range from 80 to 137 ) following RP.

Tissue microarrays. Paraffin-embedded sections obtained from the RP specimens were reviewed and mapped. Tissue microarrays were built using a manual tissue arrayer (Beecher Instruments, Alphelys). Areas representative of the tumor with the highest Gleason score were circled. For each patient, four

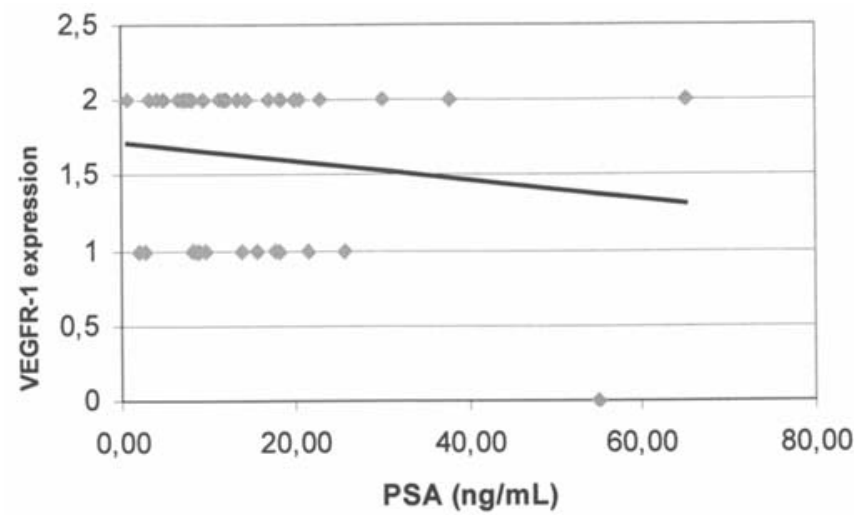

Figure 2. Intensity of VEGFR-1 expression according to PSA values.

cores of $0.6 \mathrm{~mm}$ were obtained from the circled areas of the tumor and transferred to a recipient paraffin block. Controls were obtained from non-malignant prostatic tissue.

Immunochemistry. Immunochemical staining was performed on the section mounted on poly-L-Lysine coated glass slides. Before incubation with primary antibodies, deparaffinised and rehydrated sections were incubated with avidin/biotin blocker (Vector Laboratories, Burlingame, USA) and the Fc receptor was blocked by human serum $(5 \%)$. A pre-treatment was performed in EDTA solution ( $\mathrm{pH}$ 6.0) in a microwave. An anti-VEGFR-1 (anti-Flt-1, AF321) (R\&D, Minneapolis, USA) was used at the dilution of 1:20 during $3 \mathrm{~h}$. After rinsing in PBS, the horse anti-goat biotinylated, secondary antibody (BA9005) (Abcys, Paris, France) was applied for $30 \mathrm{~min}$. A peroxydase complex was used (PK-6100). To visualise the reaction, sections were incubated with DAB substrate chromogen. Slides were mounted with Glycergel (Dakocytomation) mounting medium and evaluated under a conventional light microscopy. Tissues from breast ductal carcinoma were used as positive controls.

Evaluation of immunostaining. Two pathologists (P.C., C.B.) blinded to clinical data independently scored the slides. The intensity of staining was scored as 0 , no detectable signal, 1, weak staining, 2, moderate staining and 3, strong staining (Fig. 1). For each patient, four cores of PCa were obtained. When the four cores showed different intensity scores, the most present score was chosen to define the final intensity score. When the two pathologists found two different final intensity scores for one patient, the highest score was considered for analysis.

Statistical analysis. Statistical analysis was performed using the Statistical Analysis System, version 8.2 (SAS Institute Inc., Cary, NC, USA). The intensity of VEGFR-1 expression was considered as a non-continuous quantitative variable. The Student's t-test was used to compare the quantitative variables (patient age and PSA values) and the Chi-square test was used to compare the qualitative variables (Gleason score, pathological stage and surgical margins) between the two groups of patients. The Spearman correlation coefficient was used to study the association between the VEGFR-1 expression and PSA values. A Chi-square test was performed 
Table I. Preoperative and postoperative characteristics of patients in the two groups.

\begin{tabular}{|c|c|c|c|}
\hline & Group $1(\mathrm{~N}=17)$ & Group $2(\mathrm{~N}=23)$ & p-value \\
\hline $\begin{array}{l}\text { Patient age } \\
\text { mean value/SDa (years) }\end{array}$ & $66.3 / 6.4$ & $64.5 / 5.7$ & 0.4 \\
\hline $\begin{array}{l}\text { Pre-RP PSA } \\
\text { mean value/SD }(\mathrm{ng} / \mathrm{ml})\end{array}$ & $18.3 / 18.9$ & $13.3 / 6.9$ & 0.2 \\
\hline \multicolumn{4}{|l|}{ Gleason score } \\
\hline $\begin{array}{r}<7 \\
7\end{array}$ & $\begin{array}{l}8 \\
4\end{array}$ & $\begin{array}{r}9 \\
10\end{array}$ & \\
\hline$>7$ & 5 & 4 & 0.5 \\
\hline \multicolumn{4}{|l|}{ Pathological stage } \\
\hline $\begin{array}{l}\text { pT2 } \\
\text { pT3 }\end{array}$ & $\begin{array}{l}9 \\
8\end{array}$ & $\begin{array}{l}13 \\
10\end{array}$ & 0.8 \\
\hline Rate of PSM ${ }^{\mathrm{b}}$ & $17.6 \%$ & $13 \%$ & 0.7 \\
\hline
\end{tabular}

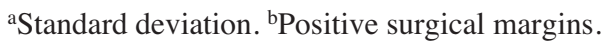

Table II. Repartition of VEGFR-1 expression according to the pathological stage of PCa.

\begin{tabular}{lcc}
\hline $\begin{array}{l}\text { Intensity score of } \\
\text { VEGFR-1 expression }\end{array}$ & pT2 stage & pT3 stage \\
N $(\%)$ & N $\%)$ \\
\hline 0 & $1(4.5 \%)$ & $0(0 \%)$ \\
1 & $12(54.6 \%)$ & $2(11.1 \%)$ \\
2 & $9(40.9 \%)$ & $15(83.3 \%)$ \\
3 & $0(0 \%)$ & $1(5.6 \%)$ \\
Total & $22(100 \%)$ & $18(100 \%)$ \\
\hline
\end{tabular}

to study the associations between the VEGFR-1 expression and other qualitative variables (Gleason score and pathological stage). Then the Chi-square test was used to compare the VEGFR-1 expression between the two groups of patients.

\section{Results}

Table I shows the preoperative and postoperative characteristics of patients in group 1 and in group 2. The age of patients, the pre-RP PSA values, the Gleason score, the pathological stages and the rates of positive surgical margins were similar in the two groups.

The intensity of VEGFR-1 expression according to pre-RP PSA values is shown in Fig. 2. There was no correlation between VEGFR-1 expression and PSA values ( $\mathrm{r}=-0.15)$. Moreover, there was no correlation between VEGFR-1 expression and the Gleason score of PCa. VEGFR-1 expression was similar in patients with low grade tumors (Gleason score $\leq 6)$ and in those with high grade tumors (Gleason score $>7)(\mathrm{p}=0.97)$.
Table II shows the repartition of VEGFR-1 expression according to the pathological stage of PCa. VEGFR-1 expression was significantly higher in pT3 tumors than in pT2 tumors $(\mathrm{p}<0.025)$.

The median intensity score of VEGFR-1 expression in the RP specimens was 2 in group 1 and 2 in group 2 . The patients who experienced cancer progression and those who remained free of recurrence following RP had the same expression of VEGFR-1.

\section{Discussion}

In our experience, the expression of VEGFR-1 in PCa did not differ between patients who experienced cancer progression and those who remained free of recurrence after RP. Our findings suggest that VEGFR-1 expression is not associated with the risk of cancer progression in patients treated for clinically-localized PCa.

Several studies have analysed the clinical impact of VEGF-A expression in PCa. Strohmeyer et al measured the expression of VEGF-A in 55 PCas and found a correlation between VEGF-A expression and tumor stage and grade (4). Furthermore, they found that the VEGF-A expression was associated with the risk of cancer progression during followup. West et al studied the expression of VEGF-A in 67 PCas, and found that the cases showing VEGF immunoreactivity in the stroma were associated with reduced survival (5). Our team previously studied the expression of VEGF-A in $100 \mathrm{RP}$ specimens (6). There was a correlation between the VEGF-A expression and the Gleason score. Another recent study, which was based on the same cohort of patients as the current work, found that the VEGF-A expression was significantly higher in patients who experienced cancer progression than in those who remained free of disease after RP (7). Therefore, there is strong evidence that suggests that VEGF-A has a prognostic value in $\mathrm{PCa}$. 
To date, three VEGF receptors have been described: VEGFR-1, VEGFR-2 and VEGFR-3. VEGF-A binds to VEGFR-1 and VEGFR-2 (1). It has been established that these receptors have the ability to stimulate angiogenesis. Nevertheless, their precise functions, and their interactions, remain to be determined. The activation and signaling of VEGFR-2 may be positively or negatively influenced by co-expression and activation of VEGFR-1.

The properties of VEGFR-1 are better known than those of VEGFR-2. VEGFR-1 participates in the recruitment of endothelial cell progenitors and activates growth factors from liver endothelial cells (8). Pathological studies show that VEGFR-1 is overexpressed in malignant prostatic tissue $(9,10)$. The expression of VEGFR-1 on the tumor cells themselves suggests a potential autocrine function for VEGF. Moreover, an aberrant methylation of the VEGFR-1 gene is often present in $\mathrm{PCa}$ (11).

Although in vivo studies suggest a role for VEGFR-1 in prostatic carcinogenesis, clinical studies regarding the impact of VEGFR-1 in patients with PCa are lacking. To our knowledge, the current study is the first to analyse the prognostic value of VEGFR-1 in patients treated for PCa. We found that VEGFR-1 expression in PCa was not associated with the risk of cancer progression after RP.

Two major limitations of our study must be recognised. Our study included a small number of patients. In group 1, we decided to include only patients who developed bone metastases after RP, which is relatively rare. Since the material available for the determination of VEGFR-1 expression was limited, we included 23 patients in the control group who did not have recurrence following intervention. Our study included only 40 patients, which may represent a limitation. On the other hand, our findings would have been probably similar with a larger study population. Indeed, the median intensity score of VEGFR-1 expression was exactly the same in the two groups. The second limitation of our study is that tissue microarrays and immunochemistry were performed retrospectively, after a long interval (more than 2 years) following RP. Our methods for the determination of VEGFR-1 expression were, therefore, not optimal. Nevertheless, a prospective evaluation seems difficult to perform. Since the wide use of PSA testing, more and more PCas are localized at the time of diagnosis (12). The stage migration of PCa and the improvements in surgical technique result in low rates of biological recurrence following RP. In the most recent series, recurrence-free survival rates are higher than $90 \%$ after several years of follow-up. A prospective study of the prognostic value of VEGFR-1 expression after RP would therefore require a very large cohort of patients.

Several studies have analysed the prognostic value of the expression of VEGF-C and its receptor, VEGFR-3, in patients with PCa. VEGF-C causes proliferation of lymphatic endothelial cells and plays a role in tumor lymphangiogenesis (13). Jennbacken et al found a higher expression of VEGF-C and VEGFR-3 in patients with lymph node metastases than in those with localized PCa (14). Li et al compared the expression of VEGFR-3 in benign prostate hyperplasia and in PCa (15). These authors found that VEGFR-3 was upregulated in cancer. Moreover, they found a correlation between VEGFR-3 expression and PSA, Gleason score and lymph node metastases.

The critical issue that remains is the therapeutic impact of VEGF and its receptors. Anti-angiogenic agents include humanised neutralising anti-VEGF monoclonal antibodies, receptor antagonists, soluble receptors, antagonistic VEGF mutants and inhibitors of VEGF receptor function (16). Antiangiogenic therapy has been shown to be effective in some variants of tumors, although its effectiveness in PCa remains to be clarified. In a recent study, human $\mathrm{CaP}$ cells were implanted into nude mouse prostates. Inhibition of the VEGF receptor induced apoptosis of tumor-associated endothelial cells, causing a second apoptotic wave of surrounding tumor cells (17). Several clinical trials investigating anti-VEGF therapy in advanced PCa are ongoing, however their results have not been published yet.

In conclusion, we found that the expression of VEGFR-1 in PCa did not differ between patients who progressed after RP and those who remained free of recurrence. These results suggest that VEGFR-1 expression in tumor tissue has no prognostic value in clinically-localized PCa. Our findings must be confirmed by larger longitudinal studies. Furthermore, the prognostic value of VEGFR-1 in advanced PCa remains to be analysed.

\section{References}

1. Hicklin DJ and Ellis LM: Role of the vascular endothelial growth factor pathway in tumor growth and angiogenesis. J Clin Oncol 23: 1011-1027, 2005.

2. Kwak C, Jin RJ, Lee C, Park MS and Lee SE: Thrombospondin-1, vascular endothelial growth factor expression and their relationship with p53 status in prostate cancer and benign prostatic hyperplasia. BJU Int 89: 303-309, 2002.

3. Jones A, Fujiyama C, Turner K, Fuggle S, Cranston D, Bicknell R and Harris AL: Elevated serum vascular endothelial growth factor in patients with hormone-escaped prostate cancer. BJU Int 85: 276-280, 2000.

4. Strohmeyer D, Rossing C, Bauerfeind A, Kaufmann O, Schlechte H, Bartsch G and Loening S: Vascular endothelial growth factor and its correlation with angiogenesis and p53 expression in prostate cancer. Prostate 45: 216-224, 2000.

5. West AF, O'Donnell M, Charlton RG, Neal DE and Leung HY: Correlation of vascular endothelial growth factor expression with fibroblast growth factor-8 expression and clinicopathologic parameters in human prostate cancer. Br J Cancer 85: 576-583, 2001.

6. Peyromaure M, Badoual C, Camparo P, Grabar S, Goulvestre C, Fulla Y, Vieillefond A, Mao K and Dinh-Xuan AT: Plasma levels and expression of vascular endothelial growth factor-A in human localized prostate cancer. Oncol Rep 18: 145-149, 2007.

7. Peyromaure M, Camparo P, Badoual C, Descazeaud A and Dinh-Xuan AT: The expression of vascular endothelial growth factor is associated with the risk of cancer progression after radical prostatectomy. BJU Int 99: 1150-1153, 2007.

8. LeCouter J, Moritz DR, Li B, Phillips GL, Liang XH, Gerber HP, Hillan KJ and Ferrara N: Angiogenesis-independent endothelial protection of liver: role of VEGFR-1. Science 299: 890-893, 2003.

9. Ferrer FA, Miller LJ, Lindquist R, Kowalczyk P, Laudone VP, Albertsen PC and Kreutzer DL: Expression of vascular endothelial growth factor receptors in human prostate cancer. Urology 54: 567-572, 1999.

10. Kollerman $\mathrm{J}$ and Helpap B: Expression of vascular endothelial growth factor (VEGF) and VEGF receptor Flk-1 in benign, premalignant, and malignant prostate tissue. Am J Clin Pathol 116: 115-121, 2001

11. Yamada Y, Watanabe M, Yamanaka M, Hirokawa Y, Suzuki H, Takagi A, Matsuzaki T, Sugimura Y, Yatani R and Shiraishi T: Aberrant methylation of the vascular endothelial growth factor receptor-1 gene in prostate cancer. Cancer Sci 94: 536-539, 2003. 
12. Aus G, Bergdahl S, Lodding P, Lilja H and Hugosson J: Prostate cancer screening decreases the absolute risk of being diagnosed with advanced prostate cancer-results from a prospective, population-based randomized controlled trial. Eur Urol 51: 659-664, 2007.

13. Trojan L, Rensch F, Voss M, Grobholz R, Weiss C, Jackson DG, Alken P and Michel MS: The role of the lymphatic system and its specific growth factor, vascular endothelial growth factor $\mathrm{C}$, for lymphogenic metastasis in prostate cancer. BJU Int 98: 903-906, 2006.

14. Jennbacken K, Vallbo C, Wang W and Damber JE: Expression of vascular endothelial growth factor C (VEGF-C) and VEGF Receptor-3 in human prostate cancer is associated with regional lymph node metastasis. Prostate 65: 110-116, 2005.
15. Li R, Younes M, Wheeler TM , Scardino P, Ohori M, Frolov A and Ayala L: Expression of vascular endothelial growth factor receptor-3 (VEGFR-3) in human prostate. Prostate 58: 193199, 2004

16. Kanda S, Miyata $\mathrm{Y}$ and Kanetake H: Current status and perspective of antiangiogenic therapy for cancer: urinary cancer. Int J Oncol 11: 90-107, 2006.

17. Busby JE, Kim SJ, Yazici S, Nakamura T, Kim JS, He J, Maya M, Wang X, Do KA, Fan D and Fidler IJ: Therapy of multidrug resistant human prostate tumors in the prostate of nude mice by simultaneous targeting of the epidermal growth factor receptor and vascular endothelial growth factor on tumor-associated endothelial cells. Prostate 66: 1788-1798, 2006. 